\title{
Políticas horizontales en la contratación pública: una taxonomía ${ }^{1-2}$
}

\author{
Sue Arrowsmith ${ }^{3}$
}

\section{RESUMEN}

La contratación pública es ampliamente utilizada para promover objetivos de naturaleza económica, ambiental y social, como el desarrollo económico de grupos sociales desfavorecidos. En este artículo se elabora una detallada taxonomía de tales políticas "horizontales". Este estudio es valioso, en primer lugar, para facilitar el análisis del fenómeno práctico de las políticas horizontales y

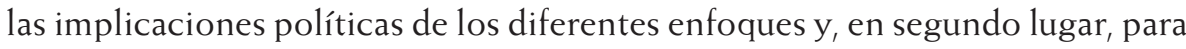

1 Titulo original "Horizontal Policies in Public Procurement: A Taxonomy", Journal of Public Procurement, 2010, vol. 10, n. ${ }^{\circ}$ 2, pp. 149-186. Traducido por Carlos Sebastián Barreto, magíster en Derecho y candidato a doctor por la Universidad de Nottingham, Notting ham, Reino Unido. Docente investigador del Departamento de Derecho Administrativo de la Universidad Externado de Colombia, Bogotá, Colombia. Correo-e: carlos.barreto@ uexternado.edu.co. Enlace ORCID: https://orcid.org/0000-0003-3418-9916.

2 Nota del traductor: Se usa "políticas horizontales" como traducción del vocablo "horizontal policies". Este artículo se basa en el capítulo 3 de S. Arrowsmith y P. KunZlik (eds.), Social and Environmental Policies in EC Procurement: New Directives and New Directions (Cambridge: CuP, 2009), que proporciona una versión un poco más detallada y matizada de la taxonomía que se expone aquí.

3 Achilles Professor de Derecho y Políticas Públicas de la Contratación Pública y directora del Grupo de Investigación y del Postgrado Ejecutivo en Contratación Pública, Universidad de Nottingham, Nottingham, Reino Unido. Sus publicaciones han sido ampliamente citadas por tribunales de todo el mundo. Es editora y fundadora de Public Procurement Law Review, y entre 2008 y 2011 lideró el proyecto con financiación europea para desarrollar la red académica global sobre regulación en contratación pública. Ha sido miembro del Grupo de Expertos en Contratación de la CNUDMI, del Grupo Asesor Internacional del Banco Mundial sobre Contratación, del Comité Asesor Independiente sobre Contratación de la Comisión Europea y actualmente es experta de la Asociación Internacional contra la Corrupción en Deporte (IPACS). Correo-e: sue.arrowsmith@nottingham.ac.uk. Fecha de recepción: 10 de mayo de 2018. Fecha de modificación: 2 de agosto de 2018. Fecha de aceptación: 5 de septiembre de 2018. Para citar el artículo: ARROWSMITH, SUE, "Políticas horizontales en la contratación pública: una taxonomía", Revista digital de Derecho Administrativo, Universidad Externado de Colombia, n. ${ }^{\circ} 21,2019$, pp. 223-261. DOI: https://doi.org/10.18601/21452946.n21.11 
esclarecer y desarrollar los marcos normativos pertinentes bajo los regímenes nacionales e internacionales. La taxonomía se basa en tres distinciones clave entre: 1) políticas limitadas a asegurar el cumplimiento de la ley, y aquellas que van más allá; 2) políticas aplicadas solamente al contrato adjudicado, y aquellas que van más allá y 3 ) nueve mecanismos diferentes por los cuales las políticas se implementan en el proceso de contratación.

Palabras clave: políticas horizontales, taxonomía, contratación estatal, políticas secundarias, políticas relacionadas con la ejecución del contrato, mecanismos para la implementación de políticas horizontales.

\section{Horizontal Policies in Public Procurement: A Taxonomy}

\section{ABSTRACT}

Public procurement is widely used to promote objectives of an economic, environmental and social nature, such as the economic development of disadvantaged social groups. This article elaborates a detailed taxonomy of such "horizontal" policies. This study is valuable, first, to facilitate analysis of the practical phenomenon of horizontal policies and of the policy implications of different approaches and, second, to illuminate and develop the relevant regulatory frameworks under national and international regimes. The taxonomy is based on three key distinctions between the following: 1. policies limited to securing compliance with legal requirements and those that go beyond such requirements ${ }_{i}$. policies applied only to the contract awarded and those that go beyond it; and 3. nine different mechanisms by which policies are implemented in the procurement process.

Keywords: Horizontal Policies, Taxonomy, Public Procurement, Secondary Policies, Policies Concerned only with the Performance of the Contract, Mechanism for Implementing Horizontal Policies.

\section{INTRODUCCIÓN}

El uso de la contratación pública como herramienta política es un fenómeno de larga data muy analizado, que cubre una amplia gama de áreas como el apoyo a condiciones laborales justas, el desarrollo regional y la provisión de oportunidades económicas para grupos desfavorecidos ${ }^{4}$. Dichas políticas a veces se

4 Véase Sue Arrowsmith, John Linarelli y Don Wallace, Regulating Public Procurement: National and International Perspectives, London: Kluwer Law International, 2000; CHRISTOPHER MCCRudden, Buying Social Justice: Equality, Government Procurement, \& Legal Change, Oxford: Oxford University Press, 2007. 
han denominado políticas "secundarias", en contraste con el llamado objetivo "primario": obtener bienes, obras o servicios en los mejores términos ${ }^{5}$, como políticas "colaterales" o como políticas socioeconómicas. Sin embargo, el término políticas "horizontales" es preferible. Este término es suficientemente genérico para abarcar todo tipo de políticas (económicas, sociales, políticas, ambientales, etc.), pero, en contraste con la terminología de políticas "secundarias" o "colaterales", no implica que estas sean necesariamente ilegítimas o subordinadas a los aspectos comerciales ${ }^{7}$.

En este artículo se elabora una taxonomía detallada de políticas horizontales independiente del objeto mismo de estas políticas. Esto es valioso por dos razones.

En primer lugar, una taxonomía detallada es útil para comprender completamente el fenómeno práctico de las políticas horizontales y las implicaciones constitucionales, y de política pública de los diferentes enfoques. Si bien el objetivo de este artículo sobre taxonomía no es ofrecer un análisis exhaustivo de las cuestiones de política pública, examinará brevemente los principales problemas que afectan el valor, la legitimidad, la transparencia y la eficacia de las políticas. Esto pone de relieve las diversas preocupaciones que surgen con diferentes enfoques y, por lo tanto, el valor de una taxonomía cuidadosamente matizada.

En segundo lugar, la taxonomía proporciona un marco para comprender y desarrollar disposiciones normativas sobre políticas horizontales. Las políticas horizontales están ampliamente reguladas tanto por las normas nacionales de contratación, como, cada vez más, por regímenes de comercio internacional como los de la Organización Mundial del Comercio (OMC) y la Unión Europea (UE), que apuntan a limitar las políticas con un impacto desproporcionado sobre el comercio ${ }^{8}$. Una taxonomía detallada puede ayudarnos a comprender el alcance preciso de la legislación, los tratados y las decisiones judiciales relevantes, y también puede facilitar un desarrollo futuro sólido al iluminar distinciones y opciones relevantes. Una vez más, este artículo centrado en la taxonomía no pretende proporcionar un análisis exhaustivo de los problemas

5 Por ejemplo: Sue Arrowsmith, The Law of Public and Utilities Procurement, London: Sweet \& Maxwell, 1996; Sue Arrowsmith, The Law of Public and Utilities Procurement, 2. a ed., London: Sweet \& Maxwell, 2005; Hans Joachim Priess y Christian Pitschas, "Secondary Criteria and Their Compatibility with EC and wTO Procurement - The Case of the German Scientology Declaration", Public Procurement Law Review, vol. 9, n. ${ }^{\circ}$ 4, London: Sweet \& Maxwell, 2000, pp. 171-195.

6 John Cibinic y Ralph NASH, Formation of Government Contracts, 3. ${ }^{\mathrm{a}}$ ed., Washington: Government Contract Program, George Washington University, 1998.

7 Sue Arrowsmith y Peter KunZliK (eds.), Social and Environmental Policies in EC procurement Law: New Directives and New Directions, Cambridge: Cambridge University Press, 2009.

8 Por ejemplo, ibíd.; Christopher McCrudden, óp. cit.; Sue Arrowsmith, Government Procurement in the World Trade Organization, London: Kluwer Law International, 2003, capítulo 13 
regulatorios. Sin embargo, dará ejemplos de cómo se tratan los diferentes enfoques en un contexto regulatorio, a fin de ilustrar la relevancia práctica de las distinciones en la taxonomía.

La taxonomía desarrolla el análisis previo del autor ${ }^{9}$, que ya se ha utilizado para los fines mencionados por otros estudiosos ${ }^{10}$. Ejemplos concretos de políticas se toman principalmente de las jurisdicciones estudiadas por el autor, especialmente la OMC, la UE, el Reino Unido, Estados Unidos y Sudáfrica. Sin embargo, los enfoques y mecanismos que ilustran se encuentran en muchas otras jurisdicciones.

\section{FUNDAMENTOS: TRES DISTINCIONES CLAVE}

La taxonomía propuesta se basa en tres distinciones clave. La primera es una distinción entre, por un lado, políticas que se limitan a garantizar el cumplimiento de la ley, por ejemplo, un requisito para que los contratistas del gobierno paguen a sus trabajadores el salario mínimo aplicable por ley a todas las empresas en la jurisdicción, y, por otro, las políticas que van más allá de esto, por ejemplo, el requisito de pagar salarios "justos" que superen el mínimo legal nacional (2).

Una segunda distinción se da entre las políticas relacionadas únicamente con la ejecución del contrato, como la obligación de pagar salarios "justos" a los empleados que participan en la ejecución del contrato, y las políticas que son más generales, como el requisito de que los contratistas paguen salarios "justos" a todos sus empleados (3).

En tercer lugar, la taxonomía distingue nueve mecanismos diferentes para la implementación de políticas horizontales, como contratos reservados, por los cuales algunos contratos están reservados únicamente para ciertos grupos y criterios de adjudicación (dando crédito a los oferentes por los beneficios ambientales o sociales de sus ofertas). Estos mecanismos implican diferentes ventajas/desventajas, incluso al equilibrar las políticas horizontales con otros objetivos, como la relación calidad-precio y la eficiencia, y son tratados de manera diferente por los regímenes de comercio debido a que inciden en varias medidas sobre el comercio $(4)^{11}$.

9 Sue Arrowsmith, John Linarelli y Don Wallace, óp. cit.; Sue Arrowsmith, The Law of Public and Utilities Procurement, $2 .^{\mathrm{a}}$ ed., óp. cit.

10 A manera de ejemplo, véase Ron B. WatermeYer, "Facilitating Sustainable Development through Public and Donor Procurement Regimes", Public Procurement Law Review, vol. 13, n. ${ }^{\circ}$ 1, London: Sweet \& Maxwell, 2004, pp. 30-55; Francis Ssennoga, "Examining Discriminatory Procurement Practices in Developing Countries", Journal of Public Procurement, vol. 6, n. ${ }^{\circ} 3$, Bingley: Emerald Publishing Limited, 2006, pp. 218-249.

11 Sue Arrowsmith, "Application of the EC Treaty and Directives to Horizontal Policies: A Critical Review", in Social and Environmental Policies in EC procurement Law: New Directives and New Directions, Cambridge: Cambridge University Press, 2009, pp. 147-248. 
Estas tres distinciones y la interrelación entre ellas serán examinadas en este artículo. Las distinciones y ciertas subdivisiones adicionales que se analizan a lo largo de este artículo se resumen en la tabla 1.

\section{TABla 1. ResUmen DE LA TAXONOMÍA}

\begin{tabular}{|c|c|}
\hline \multicolumn{2}{|r|}{ Distinción clave 1: Si se limita al cumplimiento del ordenamiento jurídico } \\
\hline 1. & Políticas que se limitan al cumplimiento de la ley \\
\hline 2. & Políticas que van más allá del cumplimiento de las normas legales generales \\
\hline \multicolumn{2}{|r|}{ Distinción clave 2: Si se limitan a la ejecución del contrato adjudicado } \\
\hline \multirow[t]{4}{*}{1.} & Políticas que se limitan a la ejecución del contrato \\
\hline & a. Decisiones de contratar o no contratar \\
\hline & b. Decisiones de qué contratar \\
\hline & $\begin{array}{l}\text { c. Mecanismos para implementar políticas en el procedimiento de adjudicación (condiciones } \\
\text { del contrato, criterios de adjudicación, etc.). } \\
\text { - Medidas de consumo } \\
\text { - Medidas de producción/ suministro } \\
\text { - Medidas de disposición final } \\
\text { - Medidas de fuerza de trabajo }\end{array}$ \\
\hline 2. & Políticas que van más allá de la ejecución del contrato \\
\hline \multicolumn{2}{|r|}{ Distinción clave 3: Mecanismos para la implementación de políticas } \\
\hline 1. & La decisión de contratar o no contratar \\
\hline 2. & La decisión de qué contratar \\
\hline 3. & Cláusulas del contrato establecidas por el contratante \\
\hline 4 . & Agrupamiento y momento de las órdenes \\
\hline 5 . & Contratos reservados \\
\hline 6. & Exclusión de los contratos por no cumplimiento con las políticas públicas \\
\hline 7. & Invitaciones preferenciales para licitar \\
\hline 8 . & Criterios de adjudicación* \\
\hline 9. & Medidas para mejorar el acceso a los contratos estatales \\
\hline
\end{tabular}

* Nota del traductor: El lector colombiano puede estar más familiarizado con los términos "criterios de selección", "criterios de evaluación" y "criterios de calificación", entre otros. Sin embargo, se mantiene el vocablo "criterio de adjudicación", término utilizado en el derecho europeo. 


\title{
2. POLÍTICAS QUE SE LIMITAN AL CUMPLIMIENTO DE LA LEY Y AQUELLAS QUE VAN MÁS ALLÁ DEL CUMPLIMIENTO DE NORMAS OBLIGATORIAS
}

\author{
2.1. Políticas Que Se limitan al Cumplimiento \\ DE OBLIGACIONES LEGALES GENERALES
}

\subsubsection{Algunos ejemplos}

Como se mencionó, una primera distinción es entre las políticas limitadas al cumplimiento de un contratista con las obligaciones legales generales, y las políticas que van más allá de esto. Un ejemplo del primer tipo de política es un compromiso contractual cuando, al llevar a cabo la ejecución del contrato, un contratista debe cumplir con la legislación general sobre salud y seguridad en el trabajo. Otra es una política seguida en muchas jurisdicciones para excluir de los contratos estatales a las empresas con condenas por ciertos delitos penales. Esto puede considerarse una política horizontal en la medida en que está dirigida a disuadir y castigar el comportamiento delictivo en lugar de limitarse a garantizar la ejecución del contrato: este es el caso, por ejemplo, de las exclusiones obligatorias por corrupción y otros delitos en virtud de las directivas de contratación de la UE ${ }^{12-13}$. Otro ejemplo interesante es la política gubernamental de Irlanda del Norte bajo la Orden de Trato Justo e Igualitario 1998 (FETO, por sus siglas en inglés) ${ }^{14}$. En un contexto histórico de divisiones religiosas y políticas entre las comunidades católica y protestante de Irlanda del Norte, este instrumento prohíbe la discriminación por motivos políticos y religiosos, y exige que las organizaciones del sector público y privado garanticen oportunidades de empleo equitativas independientemente de creencias religiosas o políticas mediante una obligación para monitorear y revisar sus prácticas de empleo y, en ciertos casos, para tomar medidas correctivas. Este régimen emplea la exclusión para celebrar contratos públicos como una sanción por el incumplimiento esa norma general.

12 Nota del traductor: Un ejemplo similar en Colombia se encuentra en la inhabilidad contemplada en el literal J del artículo 8 de la Ley 80 de 1993, modificado por la Ley 1778 de 2016. Cuando la autora usa el término "exclusion", el lector debe tener en cuenta que en el contexto colombiano la figura más similar es la inhabilidad mencionada. El uso de la palabra "exclusión" en vez de "inhabilidad" se debe a que las directivas europeas sobre contratación usan el vocablo "exclusión", y a que las exclusiones guardan algunas diferencias con las inhabilidades colombiana, como la posibilidad de excepcionarlas.

13 Sope Williams, "Co-ordinating public procurement to support EU objectives- a First Step? The Case of Exclusions for Serious Criminal Offences", in Social and Environmental Policies in EC procurement Law: New Directives and New Directions, Cambridge: Cambridge University Press, 2009, pp. 479-498.

14 Christopher McCrudden, óp. cit., pp. 305-310. 


\subsubsection{Relación con la segunda y tercera distinción}

Respecto de la segunda distinción entre las políticas relacionadas con el contrato (como se analiza más adelante) y las políticas que van más allá del contrato, las políticas limitadas a hacer cumplir las normas legales generales pueden caer dentro de cualquier categoría. Por lo tanto, una obligación contractual de cumplir la legislación de salud y seguridad cuando se ejecuta el contrato, por ejemplo, se limita claramente al contrato. Por otro lado, una exclusión obligatoria de todos los contratistas condenados por corrupción potencialmente afecta cualquier actividad comercial de los contratistas. También lo hace la política del gobierno de Irlanda del Norte bajo FETO.

En relación con los nueve mecanismos de política desarrollados en la tercera distinción, aunque muchos son potencialmente aplicables para garantizar el cumplimiento de normas legales generales, no todos son adecuados para este propósito. Este es el caso de los criterios de adjudicación del contrato. Es poco probable, por ejemplo, que una entidad otorgue puntos adicionales en la evaluación a firmas que no tienen condenas por corrupción, al tiempo que admite, aunque con una sanción en la evaluación, a firmas que sí lo hacen. Por el contrario, es probable que prefiera un enfoque que implica una obligación contractual para el cumplimiento de la ley, o la exclusión por incumplimientos pasados.

\subsubsection{Razones para usar la contratación para reforzar el cumplimiento de la ley}

¿Por qué los gobiernos adoptan políticas de contratación dirigidas meramente al cumplimiento de normas legales preexistentes? Se pueden identificar varios motivos.

Una primera razón para adoptar tales políticas es simplemente evitar asociar al gobierno con un comportamiento ilegal, tanto para dar ejemplo como para evitar las críticas del público.

Un segundo conjunto de razones para justificar las políticas que refuerzan el cumplimiento del ordenamiento jurídico es proporcionar herramientas de cumplimiento adicional para asegurar la observancia de la ley general y/o castigar las violaciones, así como reducir el riesgo de violaciones de la ley general durante la ejecución del contrato.

La posibilidad de rescindir el contrato, por ejemplo, puede inducir al cumplimiento de la ley durante la ejecución del contrato de manera más efectiva que una amenaza remota de enjuiciamiento penal. Las sanciones contractuales pueden ser especialmente útiles si la ejecución se lleva a cabo en otra jurisdicción (por ejemplo, en el caso de bienes fabricados en el extranjero) y el gobierno que adjudica el contrato tiene preocupaciones sobre la aplicación 
inadecuada de la ley en esa jurisdicción (por ejemplo, estándares en las fábricas). Las medidas para garantizar el cumplimiento de la ley por estos dos motivos a menudo se toman en relación con asuntos dentro del área de actividad de la propia entidad contratante. Por ejemplo, un Ministerio para el Medio Ambiente puede estar particularmente preocupado de no tratar con contratistas que violan la legislación ambiental.

En tercer lugar, las medidas dirigidas al cumplimiento del ordenamiento pueden estar relacionadas con garantizar la igualdad de condiciones. Por ejemplo, las empresas que no cumplen con sus obligaciones legales pagando impuestos o cumpliendo con la legislación laboral, etc., disfrutan de una ventaja competitiva desleal y pueden expulsar a los operadores legítimos del mercado. Las medidas para abordar este problema han sido importantes en las recientes reformas de la contratación pública en Sudáfrica ${ }^{15}$. Esta es también una de las razones que justifica la existencia de las disposiciones de la UE que permiten a los Estados excluir a los contratistas que no han pagado impuestos o contribuciones a la seguridad social ${ }^{16}$.

Finalmente, las medidas de contratación relacionadas con el cumplimiento por parte del contratista de los estándares legales pueden ayudar a asegurar que los fondos públicos no se utilicen para apoyar a empresas que usan contratos estatales como un medio para recaudar fondos destinados a actividades terroristas u otras actividades de tipo delictivo. Este ha sido un objetivo de las políticas de exclusión adoptadas por Nueva York ${ }^{17}$.

\subsubsection{Cuestiones procedimentales: transparencia y justicia}

En la mayoría de los países, una característica de las normas externas que son respaldadas por las medidas al interior del régimen de contratación es que existen mecanismos para juzgar su cumplimiento que son formales y transparentes, y que siguen procedimientos justos. Por ejemplo, el cumplimiento del ordenamiento jurídico puede ser juzgado por tribunales penales o agencias reguladoras. Las medidas al interior de la contratación implementadas por incumplimientos del ordenamiento pueden basarse en estos mecanismos externos, pues la aplicación de medidas en la contratación, como las exclusiones, está sujeta a condenas penales u otras determinaciones formales de incumplimiento.

15 Deen Letchmiah, "The Process of Public Sector Procurement in South Africa", Public Procurement Law Review, vol. 8, n. ${ }^{\circ}$ 1, London: Sweet \& Maxwell, 1999, pp. 15-22.

16 Así se afirma en las conclusiones del abogado general Poiares Madura en La Cascina: asuntos acumulados C-226/04-C-228 / 04, La Cascina / Ministero della Difesa [2006] ECR I-1347, párrafo 24 de las conclusiones.

17 Frank Anechiarico y James Jacobs, "Purging Corruption from Public Contracting: The Solutions are Now Part of the Problem", New York Law School Law Review, vol. 40, New York: New York Law School, 1995, p.p. 143-176. 
Este es el caso, por ejemplo, de las disposiciones de exclusión obligatorias para la corrupción en las directivas de contratación de la UE, que se aplican solo cuando existe una condena ${ }^{18}$.

Confiar en la adopción de decisiones particulares por parte de terceros (external adjudication), tribunales o agencias, puede, en cierta medida, contrarrestar las objeciones sobre la imparcialidad de los procedimientos y el posible abuso de la discreción que puede existir si la propia entidad estatal tiene que decidir si un contratista ha violado la ley. También puede limitar la necesidad de que la entidad contratante, sin la experiencia o los recursos necesarios, investigue las circunstancias de un contratista. Sin embargo, aún pueden surgir dificultades administrativas debido a no es fácil obtener pruebas de las condenas, especialmente cuando se trata de un mercado cada vez más global con empresas que pueden tener condenas en el extranjero. Las políticas basadas en condenas penales también pueden ser extremadamente difíciles de aplicar si también implican la exclusión de personas relacionadas (como empresas matrices o directores) y no existe un mecanismo externo formal para identificar a estas empresas. Esto obstaculiza la contratación eficiente y la eficacia de las políticas horizontales mismas ${ }^{19}$. Para superar estos obstáculos se necesitan mecanismos formales de información. En Irlanda del Norte, por ejemplo, la Comisión de Igualdad tiene la responsabilidad de llevar a las entidades contratantes las violaciones de FETO que resulten en exclusiones para el infractor y firmas relacionadas (artículo 62 (3) del FETO).

Las políticas en la contratación relacionadas con la violación de normas generales también se aplican a veces sin una determinación de incumplimiento bajo la legislación general. Por ejemplo, las reglas del Banco Mundial aplicables a los contratos financiados por el Banco ahora prevén ${ }^{20}$ la exclusión de los contratistas que se han visto involucrados en corrupción, incluso sin una condena penal ${ }^{21}$. Esto plantea la pregunta de si es apropiado determinar el incumplimiento e imponer sanciones sin las garantías de un proceso "normal" como un juicio penal. En gran medida, los problemas son los mismos aquí que con cualquier determinación de contratación que implique consecuencias para los contratistas, por ejemplo, la falta de calificaciones técnicas para ejecutar el contrato; es decir, cómo equilibrar los intereses de los contratistas con un

18 Sope Williams, "Co-ordinating public procurement to support EU objectives- a First Step? The Case of Exclusions for Serious Criminal Offences", in Social and Environmental Policies in EC procurement Law: New Directives and New Directions, Cambridge: Cambridge University Press, 2009, pp. 479-498.

19 Frank ANEChiarico y James JaCobs, óp. cit., pp. 143-176.

20 Nota del traductor: Se pone de presente al lector que las guías del Banco de 2004 han sido completamente reemplazadas por las Regulaciones publicadas en 2016.

21 World BANK, Guidelines for Procurement under IBRD Loans and IDA Credits, 2004, sección 1.1.5. Disponible en línea www.worldbank.org. 
proceso de contratación eficiente. Sin embargo, para las políticas basadas en el incumplimiento de las normas externas, una dimensión adicional es que la determinación del incumplimiento puede ser estigmatizante porque la conducta también está condenada por el sistema normativo "externo" (como el derecho penal). Sin embargo, esto ocurre en muchas otras situaciones, como cuando la responsabilidad civil (como la obligación de pagar una indemnización) se impone por una conducta que también es penalmente reprochable, y esto parece inobjetable por el simple hecho de que existe una dimensión adicional. La determinación de una violación no implica las mismas consecuencias que una condena (u otro procedimiento regulatorio) y, por lo tanto, no existe una razón prima facie para aplicar las reglas procesales de la ley penal.

\subsubsection{Alcances para los regímenes de comercio internacional}

Se puede observar que las políticas limitadas al cumplimiento de la ley tienen menos probabilidades de violar los regímenes de comercio internacional en materia de contratación que las que van más allá. Por ejemplo, según la legislación de la UE, los Estados miembros pueden excluir de contratos públicos a las empresas con condenas penales, pero no se les permite, por regla general, excluir a las empresas por incumplimiento de políticas gubernamentales no incluidas en la legislación ${ }^{22}$. Una razón para esto puede ser que las exclusiones basadas en normas que se establecen y aplican externamente son menos propensas al abuso que otras exclusiones, una consideración que también hace que las exclusiones anteriores sean potencialmente más aceptables bajo las normas nacionales.

En general, las normas legales aplicables para ejecutar el contrato que se refuerzan a través de las medidas en la contratación serán las normas del propio estado adjudicador: la mayoría de los licitantes serán contratistas nacionales sujetos a la legislación nacional. Sin embargo, cuando los contratos son realizados por contratistas extranjeros y/o parte del objeto del contrato (como la fabricación de bienes) se lleva a cabo en el exterior, pueden aplicarse normas legales establecidas por otro Estado -como el Estado donde se ejecuta parte del contrato-y estas normas pueden también ser objeto de medidas en la contratación por parte del Estado que adjudica el contrato. De hecho, como se mencionó anteriormente, los gobiernos pueden encontrar que las sanciones al interior del régimen de contratación son especialmente útiles cuando otros métodos de ejecución de la ley, como las sanciones penales, están fuera de su propio control. Los Estados también pueden desear adoptar políticas que tengan en cuenta el cumplimiento de las leyes de otros países, más

22 Sue Arrowsmith, "Application of the EC Treaty and Directives to Horizontal Policies: a Critical Review", óp. cit., pp. 147-248. 
allá del contrato, por ejemplo, exclusiones por corrupción independientemente del país de condena.

\subsection{Políticas Que Van más allá del Cumplimiento De la ley}

\subsubsection{Algunos ejemplos y la relación con la segunda y tercera distinción}

Adicionalmente, las políticas horizontales pueden proporcionar beneficios sociales o ambientales que van más allá de los que surgen del cumplimiento de los contratistas con las normas generales.

Nuevamente, tales políticas pueden estar limitadas a funcionar dentro el contrato mismo, por ejemplo, una obligación para que un contratista contrate a una cierta proporción de personas con discapacidad en la ejecución del objeto contractual. Sin embargo, también pueden extenderse más allá. Para promover la igualdad racial y de género, un gobierno podría excluir a las empresas que no adopten una política proactiva para implementar un equilibrio étnico y de género en su fuerza de trabajo, aunque tal requisito no se imponga a las empresas en general. Tal política ha sido adoptada por el gobierno de Estados Unidos, por ejemplo, bajo un programa que se extiende también a otras áreas, como el estatus de veterano ${ }^{23}$. Este enfoque contrasta con el uso de la contratación para promover la igualdad política y religiosa en Irlanda del Norte, donde las sanciones en la contratación respaldan obligaciones legales generales aplicables a todas las empresas en Irlanda del Norte, no solo a los contratistas del gobierno.

\subsubsection{Cuestiones constitucionales}

En algunas ocasiones, las políticas limitadas a contratistas del gobierno se establecen sin ninguna base legislativa. En efecto, el hecho de que la legislación puede no ser necesaria, pero se requiere para enfoques alternativos, como las sanciones penales, o se necesita solo en una forma particular-como regulación en lugar de legislación- puede ser una de las razones para escoger la contratación como herramienta de política pública. La decisión del Gobierno de Estados Unidos de implementar políticas de igualdad a través de la contratación parece haberse tomado debido a la dificultad de implementar legislación en el Congreso ${ }^{24}$. La contratación también puede elegirse porque es la única herramienta disponible. Las autoridades locales en el Reino Unido, en la década de 1980, utilizaron la contratación para apoyar diversas políticas, 
como la igualdad de género ${ }^{25}$, en virtud de que una regulación más directa estaba claramente más allá de sus poderes legales.

Esto a veces ha planteado preocupaciones constitucionales en torno de si es apropiado que las autoridades hagan indirectamente lo que no pueden hacer directamente. En algunos casos, los tribunales o el legislador han intervenido para aclarar u ordenar que dicha "regulación indirecta" está, de hecho, prohibida. Este fue el caso con el uso de la contratación pública por parte de las autoridades locales del Reino Unido para apoyar la política contra el apartheid en la década de 1980, que excluía de los procedimientos de selección a empresas que tenían relaciones con Sudáfrica ${ }^{26}$.

Hasta cierto punto, este enfoque "horizontal" para la formulación de políticas ha sido respaldado de manera positiva por la legislación, que ha "incorporado" (mainstreaming) ciertas políticas en todas las actividades del gobierno. La legislación del Reino Unido ahora requiere que todas las autoridades públicas consideren varios asuntos de igualdad en el ejercicio de todas sus funciones, incluida la función de contratar, aunque la mayoría de las autoridades no tienen poderes regulatorios sobre esta materia (actualmente bajo Race Relations Act 1976, s. 71, Sex Discrimination Act 1975, s. 76A y Disability Discrimination Act 1995, s. 49A), y en virtud de Equality Act 2010, s. 149, que cubre una amplia gama de características (entre ellas, género, edad, embarazo y maternidad, raza, religión o creencia, sexo, orientación sexual y personas con habilidades especiales).

Debido a que las políticas horizontales a menudo aparecen como una forma de regulación, ces justificable utilizar la contratación de esta manera cuando el efecto es "regular" la conducta de los contratistas del Estado, pero no a otras empresas? Mientras que las personas de derecho privado generalmente no tienen otra opción que expresar sus preferencias sociales y ambientales a través de compras y actividades similares, y cada vez lo hacen como un aspecto de la responsabilidad social empresarial ${ }^{27}$, el Estado tiene la opción de adoptar medidas regulatorias más generales.

Se pueden ofrecer dos justificaciones para este uso de la contratación pública como mecanismo de regulación. Una es asegurar que el gobierno esté asociado con los más altos estándares posibles, al igual que con las políticas diseñadas para garantizar el cumplimiento legal se pueden adoptar medidas horizontales en la contratación tanto para dar un ejemplo -que puede alentar una mayor aceptación de las normas-, como para evitar las críticas del público.

25 John Carr, New Roads to Equality: Contract Compliance for the United Kingdom?, London: The Fabian Society, 1987.

26 Véase R v Lewisham LBC, ex P. Shell UK [1988] 1 All ER 938 y Local Government Act 1988 , s. 17.

27 David Vogel, The Market for Virtue: The Potential and Limits of Corporate Social Responsibility, Washington: Brookings Institution Press, 2005, capítulo 4. 
La segunda justificación tiene que ver con la efectividad: la contratación es, en algunos campos, un instrumento de política más eficaz que las alternativas, como las sanciones penales, y esto puede justificar la decisión de concentrar recursos limitados en la alternativa más eficaz. Como Morris afirma (en el contexto de las políticas de igualdad de oportunidades):

el modelo individual de recursos y decisión particular (adjudication) para enfrentar la discriminación está fundamentalmente viciado por problemas de legalismo, procedimientos tortuosos y satisfacción de la carga de la prueba. El cumplimiento del contrato, por el contrario, no se ve obstaculizado por estos problemas. Evita las deficiencias inherentes a las decisiones individuales (adjudication) o la investigación institucional ${ }^{28}$.

Además, la estrecha relación del gobierno con sus contratistas puede ayudar a garantizar una supervisión efectiva de las políticas.

El uso de la contratación de una manera que va más allá de exigir el simple cumplimiento de la ley también puede plantear otras inquietudes constitucionales, especialmente -como destacó Daintith ${ }^{29}$ - cuando la contratación refuerza la aplicación de estándares normativos a comportamientos que no se relacionan con la ejecución del contrato. Tales preocupaciones pueden estar relacionadas, por ejemplo, con la legitimidad democrática de la regulación a través de la contratación, la adecuación de las garantías procesales, la seguridad jurídica y la transparencia.

Como se mostró, pueden surgir preocupaciones similares con las políticas limitadas al cumplimiento de la ley, por ejemplo, sobre las garantías procesales cuando una entidad contratante toma su propia decisión en relación con la violación de la ley por parte de un contratista. Sin embargo, tales preocupaciones son más frecuentes con políticas que van más allá del cumplimiento del ordenamiento jurídico.

Una razón para esto es que, como se mencionó, la regulación a través de la contratación puede no requerir legislación, que tiende a proporcionar de mejor manera la aplicación de los principios constitucionales a los procedimientos administrativos. Un segundo factor relacionado es que (como ya se mencionó) cuando la contratación se utiliza meramente para hacer cumplir la ley, los mecanismos externos, como los tribunales penales, a menudo se utilizan para determinar el cumplimiento, y estos proporcionan garantías para los contratistas. Una tercera consideración es que las contrataciones del gobierno, algunas veces, no están sujetas a controles constitucionales adecuados

28 Peter Morris, "Legal Regulation of Contract Compliance: an Anglo-American Comparison", Anglo-American Law Review, vol. 19, n. ${ }^{\circ}$ 2, Chichester: Justice of Peace, 1990, pp. 88-89.

29 Terence Daintith, "Regulation by Contract: The New Prerogative", Current Legal Problems, vol. 32, Oxford: Oxford University Press, 1979, pp. 41-64. 
o claros, por ejemplo, los principios del derecho administrativo general con respecto al debido proceso y la racionalidad en la toma de decisiones ${ }^{30-31}$. Sin embargo, estas preocupaciones no arrojan ninguna duda sobre la idoneidad de la contratación como herramienta de política per se, sino simplemente sobre la forma en que los principios constitucionales se aplican en la práctica a la "regulación a través de la contratación". Sencillamente se resalta la necesidad de tener especial cuidado para implementar tales políticas de acuerdo con los valores y principios constitucionales.

En la práctica, muchos regímenes toman medidas para hacer esto y usan instrumentos formales -incluida la legislación- para implementar políticas horizontales, incluso cuando no son requeridas constitucionalmente. A menudo establecen la política en detalle para garantizar la seguridad jurídica, la transparencia y el "debido proceso" para salvaguardar los intereses de los contratistas.

Como se mencionó, una opción es asignar a un cuerpo externo a las entidades contratantes la toma de decisiones, tales como si los contratistas han violado las normas no-legales aplicables. Un ejemplo es la Oficina de Programas de Cumplimiento de Contratos Federales de Estados Unidos (United States Office of Federal Contract Compliance Programs), parte de la Administración de Estándares de Trabajo del Departamento de Trabajo de Estados Unidos (US Department of Labor's Employment Standards Administration). Esta oficina tiene la responsabilidad central de los programas sobre acciones afirmativas en el lugar de trabajo, incluidas las decisiones sobre incumplimiento por parte de los contratistas. La responsabilidad centralizada de realizar, cotejar y publicitar las decisiones de los programas horizontales puede ayudar a promover la transparencia, la coherencia y la aplicación efectiva, también puede dar las garantías adecuadas para los contratistas, concentrando los recursos y la experiencia en un solo lugar, y puede ayudar a evitar los retrasos en la contratación, retrasos que pueden ocurrir cuando las decisiones se toman ad boc. Esto es particularmente relevante para decisiones complejas, como la forma en que el incumplimiento por parte de una empresa debería afectar a las empresas asociadas.

\subsubsection{Implicaciones para los regímenes de comercio internacional}

Por último, se puede señalar un punto de creciente importancia en la era de la globalización, a saber, que la participación extranjera crea complejidades

30 Stephen Balley, "Judicial Review of Contracting Decisions", Public Law, n. ${ }^{\circ}$ 3, London: Sweet \& Maxwel, 2000, pp. 444-463.

31 Nota del traductor: Este es un comentario que no aplica para el caso colombiano ni resulta relevante para el mismo, pues en esta jurisdicción el derecho administrativo y todos sus estándares aplican con pleno rigor en la contratación estatal. Esto es así a diferencia del caso británico, en donde la aplicación de los principios de derecho administrativo a la contratación estatal es bastante limitada. 
adicionales significativas para las políticas que van más allá del cumplimiento del ordenamiento jurídico.

En relación con ello, los gobiernos que implementan tales políticas sociales deben decidir si aplicarlas a empresas extranjeras y/o al trabajo realizado en el extranjero. Cuando los gobiernos intentan "comprar" a través de la contratación estatal beneficios sociales, tales como capacitación (exigiendo que los contratistas capaciten a los trabajadores que participan en la ejecución del contrato) u oportunidades de empleo para personas con discapacidades, es posible que no deseen pagarlos cuando los beneficios se salgan de su propia jurisdicción. También puede parecer inapropiado, además de involucrar costos injustificados, utilizar la contratación para "regular" empresas o actividades que generalmente están por fuera de su jurisdicción, por ejemplo, para garantizar el cumplimiento de los programas para crear un lugar de trabajo libre de drogas.

Una posibilidad aquí es que los gobiernos limiten sus medidas a contratistas/ actividades nacionales. Sin embargo, en los contratos que involucran competencia extranjera (ya sea bajo regímenes internacionales de libre comercio o de otra manera), esto puede conllevar dos cosas. En primer lugar, que se pierda la oportunidad de utilizar la contratación como una herramienta de política (si el contrato es adjudicado a una empresa extranjera o si se subcontrata off-shore), $y$, en segundo lugar, que las empresas nacionales se pongan en una desventaja competitiva (debido a los costos adicionales de cumplir con la política, que es una desventaja más visible e inmediata que la desventaja que surge de cumplir con una legislación o reglamentación más general).

Otra posibilidad es excluir empresas/actividades extranjeras por completo. Sin embargo, cuando el contrato está regulado por normas comerciales -como el Acuerdo sobre Contratación Pública de la Organización Mundial del Comercio (ACP)-, esto puede requerir la negociación de excepciones o el uso de derogación a los tratados que son de alcance incierto.

Estos temas se están volviendo más prominentes con el aumento de la globalización y la expansión de la cobertura de los acuerdos comerciales sobre contratación. Cómo equilibrar los intereses del gobierno al utilizar la contratación para implementar políticas horizontales con consideraciones de libre comercio es, de hecho, uno de los temas más urgentes y debatidos planteados por los acuerdos comerciales en materia de contratación ${ }^{32}$. Esto es particularmente cierto en el caso de las políticas que van más allá del cumplimiento legal y que van más allá del contrato (ver a continuación), que pueden tener un impacto significativo en el comercio. 


\section{POLÍTICAS LIMITADAS A LA EJECUCIÓN DEL CONTRATO QUE SE ADJUDICA Y POLÍTICAS QUE VAN MÁS ALLÁ DE LA EJECUCIÓN CONTRACTUAL}

La segunda distinción en la taxonomía es entre las políticas que se refieren solo a la ejecución del contrato adjudicado, y las políticas que se extienden a otras actividades del contratista. Esto se caracteriza a veces como una distinción entre el "gobierno como comprador" y el "gobierno como regulador" ${ }^{\text {"33. Ade- }}$ más, surgen varias preocupaciones de política que a menudo se corresponden ampliamente con estos dos grupos de medidas.

Sin embargo, tanto para describir y evaluar las políticas horizontales como para analizar el impacto de las normas legales, esta caracterización simple es muy simplista y se necesita una taxonomía más minuciosa como se realiza a continuación $n^{34}$.

\subsection{Políticas confinadas a la ejecución del contrato}

Las políticas relacionadas con el contrato pueden buscar simplemente garantizar el cumplimiento de la ley, por ejemplo, que el contratista cumpla con las leyes de salud y seguridad al ejecutar el contrato. Sin embargo, también pueden buscar beneficios que van más allá de los previstos por la ley, como el empleo de personas con discapacidad en la ejecución del contrato por encima de los requisitos mínimos legales.

Las políticas dentro de este grupo se pueden subdividir en varias categorías adicionales que son útiles para el análisis legal. Las das primeras se corresponden con los dos primeros mecanismos para implementar políticas horizontales que se detallan a continuación, mientras que las otras son subdivisiones relevantes para todos o algunos de los mecanismos restantes (condiciones del contrato, criterios de adjudicación, exclusiones, etc.). Ellas son las siguientes:

1. Decisión de contratar o no contratar: Por ejemplo, una decisión de no continuar con las obras de construcción debido al impacto en el medio ambiente.

2. Decisión sobre qué contratar: Por ejemplo, para comprar helicópteros en lugar de botes salvavidas para el rescate marítimo (ver más adelante).

3. Políticas implementadas a través de mecanismos en el procedimiento de selección: Este tercer grupo se puede dividir en cuatro subgrupos:

a. Medidas de consumo: Esto se refiere al efecto de productos, obras o servicios cuando son consumidos, ya sea por la entidad contratante, el público u otros. Por ejemplo, el gobierno puede exigir que todos los empleados o miembros

33 Por ejemplo: Christopher McCrudden, óp. cit.; Sue Arrowsmith, The Law of Public and Utilities Procurement, 2. ${ }^{a}$ ed., óp. cit.

34 Sue Arrowsmith y Peter Kunzlik, óp. cit. 
del público puedan utilizar el objeto particular del contrato; que los alimentos servidos en comedores gubernamentales o en las escuelas sean aptos para el consumo de cualquier grupo religioso, o que los equipos de tecnología en la oficinas y bibliotecas sean accesibles para personas con discapacidad ${ }^{35}$.

b. Medidas de producción y suministro: Comprende todas las medidas que se refieren al impacto de la producción o suministro (pero excluye las cuestiones relativas a la mano de obra contratada, tema que se trata por separado). La producción y el suministro están incluidos en una categoría, puesto que, en el caso de ciertas obras y servicios, la producción y el suministro al cliente se combinan (aunque con la adquisición de bienes generalmente se trata de procesos separados). Esta subcategoría es particularmente importante para la política ambiental. Las entidades podrían especificar que los productos deben ser producidos con materiales reciclados, o deben obtenerse de fuentes sostenibles, por ejemplo, electricidad de fuentes renovables ${ }^{36}$ o madera y productos derivados de fuentes legales y sostenibles (esta es la política del gobierno del Reino Unido, Departamento de Medio Ambiente, Alimentación y Asuntos Rurales $^{37}$ ). Para obras o servicios, las autoridades podrían tratar de limitar el impacto ambiental, por ejemplo, mediante la obligación de no desperdiciar energía durante la construcción o prestación del servicio. Las consideraciones sociales pueden implementarse a través de obligaciones con respecto a la ubicación de la producción (por ejemplo, para bienes producidos localmente) o la forma de producción, así, el gobierno sudafricano utiliza métodos de construcción intensivos en mano de obra, como caminos rurales de grava, para proporcionar oportunidades de empleo ${ }^{38}$.

c. Medidas de disposición final: En tercer lugar, los gobiernos pueden incluir clausulas sobre la disposición final de bienes, tales como la obligación para

35 Rosemary Boyle, "Disability Issues in Procurement", in Social and Environmental Policies in EC procurement Law: New Directives and New Directions, Cambridge: Cambridge University Press, 2009, pp. 310-344; Christopher Yukins, "Making Federal Information Technology Accessible: A Case Study in Social Policy and Procurement", Public Contract Law Journal, vol. 33, n. ${ }^{\circ}$ 4, Washington: American Bar - George Washington University Law School, 2004, pp. 667-724.

36 Peter Kunzlik, "The Procurement of 'Green' Energy", in Social and Environmental Policies in EC procurement Law: New Directives and New Directions), Cambridge: Cambridge University Press, 2009, pp. 369-407

37 Department for Environment, Food and Rural Affairs, UK Government Sustainable Development Action Plan (November 2007 - November 2009), 2007, p. 29. Disponible en línea http://www.defra.gov.uk/sustainable/government/publications/pdf/SustainableProcurementActionPlan.pdf.

38 Government of South Africa, Green Paper on Public Sector Procurement (GN n. ${ }^{\circ} 691$, GG17928 of 14 April 1997); UNCITRaL, UNCITRal Model Law on Procurement of Goods, Construction and Services, 1997, pp. 61-65. Disponible en línea http://www.uncitral.org/ uncitral/en/uncitral_texts/procurement_infrastructure/1994Model.html. 
que los contratistas reciclen los productos suministrados después de que estos han sido utilizados.

d. Medidas sobre el personal: Finalmente, los gobiernos pueden implementar políticas horizontales a través de medidas relacionadas con la composición o condiciones de trabajo del personal contratado. Al igual que otras medidas en la contratación, estas pueden limitarse a garantizar el cumplimiento de las obligaciones legales, pero a menudo van más allá. En el Reino Unido, durante la primera parte del siglo XX, el gobierno central insertó términos contractuales que exigían condiciones de trabajo "justas" para los empleados en contratos gubernamentales que eran más favorables que aquellos bajo la ley general ${ }^{39}$. Las cláusulas contractuales también pueden obligar a que los contratistas brinden trabajo en el contrato para personas con discapacidades.

Las políticas pueden estar relacionadas con más de uno de estos grupos, por ejemplo, un criterio de adjudicación medioambiental que tenga en cuenta la huella de carbono total de un producto. Sin embargo, es útil distinguir los cuatro subgrupos tanto para propósitos de exposición, como debido a que existe un tratamiento diferente en el discurso legal y político. Por ejemplo, existe un importante debate en el contexto de acuerdos comerciales, incluso dentro de la OMC y la UE, sobre si las medidas relacionadas con los impactos de la producción son o deberían tratarse de manera distinta a las medidas relacionadas con el consumo, especialmente con medidas de producción incluidas en los contratos de suministro de bienes ${ }^{40}$. Una razón para esto es el mayor impacto de las medidas de producción en el comercio: en la práctica, las medidas de producción implementadas para los bienes suministrados al gobierno pueden necesitar ser aplicadas en toda una fábrica o negocio como un todo, y así pueden desincentivar la participación en la contratación estatal. Otro motivo de preocupación sobre la producción mide el posible efecto extrajurisdiccional, pues la producción regulada puede tener lugar en el exterior. En estos aspectos, las medidas de producción centradas en la ejecución del contrato, son, de hecho, similares en sus impactos a las políticas que van más allá del contrato, como se analiza en la siguiente sección.

\subsection{Políticas que van más allá de la ejecución contractual}

Con fines eminentemente descriptivos, las políticas que van más allá del contrato pueden dividirse en tres grupos principales. in Public Procurement: Global Revolution, London: Kluwer Law International, 1998, pp. 199218; Sue Arrowsmith y Peter KunZlik, óp. cit., de manera más general sobre el tratamiento diferente de estas subcategorías en la legislación de la UE. 
En el primer grupo se pueden incluir políticas que regulan el comportamiento de los contratistas en toda su actividad comercial en conjunto. Los ejemplos ya mencionados son políticas en Estados Unidos e Irlanda del Norte (bajo FETO), que excluyen de los contratos gubernamentales a las empresas que no desarrollan políticas de acciones afirmativas para la igualdad laboral. Otros ejemplos son las medidas que excluyen a las empresas que tienen relaciones comerciales con países "indeseables". Una ilustración notable de este tipo de medida es la antigua política de algunos estados de Estados Unidos de excluir a los contratistas que tenían conexiones con Myanmar ${ }^{41}$, declarada inconstitucional bajo la ley estadounidense ${ }^{42}$ e impugnada en el marco del $\mathrm{ACP}^{43}$. De nuevo, algunas políticas de este tipo se limitan al cumplimiento de la ley, a garantizar que el contratista cumpla con la ley en sus actividades, como con la política de Irlanda del Norte bajo FETO. Sin embargo, otros imponen estándares a los contratistas más allá de aquellos con los que deben cumplir las empresas en general, como ocurre con la política federal de igualdad de Estados Unidos.

Un segundo grupo que va más allá del marco de la ejecución del contrato comprende políticas bajo las cuales el gobierno no trata de cambiar el comportamiento de los contratistas, sino de apoyar empresas con características particulares. Algunos ejemplos son las preferencias de adjudicación o los contratos reservados para ayudar a las pequeñas empresas y/o grupos sociales desfavorecidos. Así, en Sudáfrica, el gobierno ha utilizado ampliamente la contratación pública para corregir las desigualdades institucionalizadas bajo el antiguo régimen del apartheid ${ }^{44}$ y respalda expresamente este uso de la contratación estatal en la Constitución. En Estados Unidos ${ }^{45}$, medidas en la contratación se han utilizado a menudo para respaldar talleres que ofrecen empleo a personas con discapacidad, y en el caso de las directivas de contratación de la UE, que permiten la existencia de contratos reservados para esos talleres ${ }^{46}$. Por supuesto, no existe una distinción clara entre el primer y el segundo grupo; por ejemplo, las políticas que respaldan a las empresas que son propiedad de grupos minoritarios pueden buscar tanto apoyar a estas empresas como alentar a las establecidas a incorporar minorías en el negocio.

Finalmente, el tercer grupo incluye offsets (compensaciones): políticas que involucran a los contratistas en la provisión de beneficios a la comunidad, por

41 Sara H. Cleveland, "Norm Internalization and U.S. Economic Sanctions", Yale Journal of International Law, vol. 26, n. ${ }^{\circ}$ 1, New Haven: Yale University, 2001, pp. 1-102. John LinareLLI, "Economic Sanctions and the US Supreme Court: Crosby v National Foreign Trade Council", Public Procurement Law Review, vol. 10, n. ${ }^{\circ}$ 3, London: Sweet \& Maxwell, 2001, pp. 91-96. 
ejemplo, mediante la construcción de instalaciones o fábricas comunitarias, que no están necesariamente relacionadas con el contrato.

Las políticas dirigidas, o que afectan, el comportamiento fuera de los contratos estatales a menudo impone una mayor carga a los contratistas que las limitadas al contrato, especialmente cuando se extienden a todas las actividades del contratista. Como resultado, también hay costos potencialmente mayores para el proceso de contratación. Estos surgen tanto por los costos de cumplimiento reflejados en las licitaciones (especialmente con las condiciones contractuales establecidas por el gobierno), como porque estas políticas pueden reducir el número de proponentes. Dichas políticas también han generado preocupaciones en el marco de las reglas de libre comercio debido a su impacto sobre el acceso al mercado: hasta el punto que en la medida en que vayan más allá del cumplimiento de la ley están, al menos, generalmente prohibidas por las directivas de contratación de la UE ${ }^{47}$.

Sin embargo, esta diferencia entre las políticas formalmente limitadas a la ejecución del contrato y las que van más allá es una cuestión de grado, que depende de la naturaleza del contrato. Por lo tanto, como hemos mencionado, incluso las políticas formalmente limitadas a la ejecución del contrato pueden tener un impacto significativo en la actividad comercial de manera más general. Por ejemplo, el cumplimiento de una cláusula que exige que se produzcan suministros sin contaminación puede exigir efectivamente a una empresa cambiar sus métodos de producción. Esto ha llevado a la Comisión Europea a sugerir, polémicamente, que bajo la legislación de la UE no se deben considerar las medidas relacionadas con la producción como medidas relacionadas con la ejecución del contrato, generalmente permitidas, sino como medidas prohibidas ${ }^{48}$.

De manera similar, puede ser difícil para un contratista hacer los cambios requeridos a las condiciones laborales solamente para los trabajadores que ejecutan contratos estatales. Lo anterior puede ocurrir porque los trabajadores podrían estar involucrados tanto en trabajos relacionados con contratos estatales como con otros contratos, o porque es problemático aplicar diferentes salarios y condiciones laborales a trabajos similares dentro de la organización.

47 Sue Arrowsmith, "Application of the EC Treaty and Directives to Horizontal Policies: a Critical Review", óp. cit., pp. 147-248.

48 European Commission, Interpretative Communication on the Community Law Applicable to Public Procurement and the Possibilities for Integrating Social Considerations into Public Procurement, (COM (2001) 566 final), 2001, p. 18. Disponible en línea http:// ec.europa.eu. 


\section{MECANISMO PARA LA IMPLEMENTACIÓN DE POLÍTICAS HORIZONTALES}

Como se dijo, una tercera distinción en la taxonomía es entre los diferentes mecanismos para implementar políticas horizontales. Muchos de estos mecanismos son apropiados para todo tipo de políticas, es decir, tanto para políticas que se limitan al cumplimiento de la ley como para aquellas que no lo hacen, y tanto para políticas que se limitan a la ejecución del contrato como para aquellas que van más allá. Sin embargo, este no es siempre el caso: la posibilidad para un contratista de cumplir con las obligaciones legales puede ser inadecuada como un criterio de adjudicación.

Cualquiera que sea el mecanismo escogido, la incorporación de políticas horizontales en la contratación estatal generalmente implica costos que deben sopesarse frente a cualquier beneficio. Hay una cantidad significativa de investigaciones que evalúa el impacto de los diferentes mecanismos de políticas ${ }^{49}$, pero queda mucho por hacer.

En primer lugar, las políticas horizontales a menudo implican pagar precios más altos y/o algún impacto adverso en otras características de la oferta de un proveedor, como la calidad del servicio.

Este no es, por supuesto, de manera invariable el caso. De hecho, algunas políticas horizontales traen beneficios comerciales. Así, la compra de bombillas de bajo consumo más caras puede generar un ahorro si el gasto inicial se ve compensado por menores gastos de energía, mientras que las políticas para mejorar el acceso de pequeños proveedores sin preferencias (mecanismo explicado más abajo) pueden conducir a una mayor competencia $\mathrm{y}$, por lo tanto, a mejores precios.

A pesar de esto, muchas políticas sí implican costos adicionales. Estos pueden surgir de los costos para los proveedores de proporcionar los beneficios sociales o ambientales (por ejemplo, los costos adicionales de las características para hacer que los autobuses sean accesibles a sillas de ruedas o de mejores condiciones de trabajo). Esos costos también pueden surgir del hecho de que algunas empresas no pueden participar, lo que reduce la competencia. La posición en este caso, en efecto, no sería diferente en principio de la que se aplica al comprar beneficios "comerciales" en un contrato, como cuando la entidad exige una alta calidad para los servicios prestados en un contrato. Sin embargo, los costos y beneficios de ciertas políticas horizontales, especialmente aquellas que regulan el comportamiento más allá del contrato, pueden ser difíciles de establecer. Se describen a continuación cómo los diferentes mecanismos de políticas pueden afectar la capacidad del gobierno tanto para identificar como para controlar el alcance de los costos y beneficios. 
Un segundo costo puede surgir de la existencia de una mayor discreción, por ejemplo, la discreción para excluir a las empresas que no cumplen con los requisitos horizontales, o la discreción adicional involucrada en la aplicación de criterios sociales en la adjudicación. Esta discreción no es necesariamente mayor que en otras evaluaciones, por ejemplo, de capacidad técnica, pero el alcance general de la discrecionalidad en la contratación puede aumentar. Esta será una gran preocupación para los sistemas que ponen énfasis en limitar la discreción y su potencial abuso como un medio para alcanzar los objetivos en la contratación, bien sea que esos objetivos sean la relación calidad-precio, reducción de la corrupción y/o prevención de la discriminación por motivos de nacionalidad. Esto varía entre Estados y entidades de acuerdo con factores tales como el grado de corrupción y las habilidades de los oficiales de contratación ${ }^{50}$.

Otro costo de las políticas horizontales se relaciona con los de verificar el cumplimiento, evaluar los criterios de adjudicación adicionales y demás, y el costo para las empresas de cumplir con requisitos administrativos adicionales (que también pueden disuadir de la participación) ${ }^{51}$. El potencial de las políticas horizontales para crear costos significativos, si se implementan de manera efectiva, queda ilustrado por los problemas encontrados en los sistemas para excluir a los contratistas involucrados en corrupción: en particular, evitar que firmas inescrupulosas evadan la exclusión estableciendo nuevas compañías puede implicar costos desproporcionados para la administración y contratistas en general $1^{52}$. La interrupción y los costos de los recursos administrativos o litigios también pueden aumentar al agregar políticas horizontales en el proceso de contratación.

Tanto los costos, como los beneficios, varían de acuerdo con el mecanismo utilizado, así como según otros factores, como la consistencia y efectividad con la que se aplica y supervisa la política. Los principales costos y beneficios inherentes de los diferentes mecanismos se señalan brevemente en la siguiente taxonomía de los mecanismos existentes.

\subsection{LA DECISIÓN DE CONTRATAR O NO CONTRATAR}

La razón misma para celebrar muchos contratos estatales es, en primer lugar, implementar políticas sociales (proporcionar instalaciones de salud, educación,

50 Véase Steve Kelman, Procurement and Public Management, Washington: aei Press, 1990; Sue Arrowsmith, "The EC Procurement Directives, National Procurement Policies and Better Governance: the Case for a New Approach", óp. cit., pp. 3-24; STEVEn SCHOOneR, "Fear of Oversight: The Fundamental Failure of Businesslike Government", American University Law Review, vol. 50, n. ${ }^{\circ}$, Washington: American University Washington College of Law, 2001, pp. 627-724.

51 Por ejemplo, Patricia Wittie, "Transnational Concerns: Domestic Preferences", Public Procurement Law Review, vol. 11, n. ${ }^{\circ}$ 2, London: Sweet \& Maxwell, 2002, pp. 145-148, quien considera el sistema de preferencias nacionales en Estados Unidos.

52 Frank Anechiarico y James Jacobs, óp. cit., pp. 143-176. 
etc.) o proporcionar beneficios ambientales (por ejemplo, con la adquisición de una planta de reciclaje). Además, la decisión de celebrar un contrato en particular puede estar influenciada por preocupaciones sociales o ambientales que son distintas de las logradas a través de los productos, obras o servicios en sí mismos, es decir, por preocupaciones horizontales.

En primer lugar, la decisión de contratar puede hacerse no solo por los beneficios de esos productos, obras o servicios directamente, sino también por otros beneficios resultantes. Por ejemplo, los Estados han emprendido a menudo programas de obras públicas para proporcionar empleo y un impulso económico en tiempos de alto desempleo o recesión, algo que muchos gobiernos consideran en el clima económico actual ${ }^{53}$. Tanto el deseo de edificios u otra infraestructura para oficinas, transporte, etc., como el impulso al empleo pueden influir en la decisión de seguir adelante con un proyecto. Un gobierno también podría emprender un programa que utilice productos innovadores no solo por el beneficio directo (para vivienda), sino también para desarrollar los productos determinados, por ejemplo, construyendo viviendas experimentales utilizando materiales ecológicos.

En segundo lugar, un gobierno podría decidir no proceder con un proyecto que de otro modo haría debido a los impactos sociales o ambientales. Por ejemplo, después de una investigación puede decidir abandonar un plan para una represa debido a un impacto ambiental adverso.

\subsection{LA DECISIÓN DE QUÉ CONTRATAR}

Suponiendo que se ha tomado la decisión de emprender un proyecto o llevar a cabo una función en particular, los medios básicos elegidos pueden estar influenciados por preocupaciones sociales o ambientales, así como por las necesidades mismas de la función o proyecto. Por ejemplo, una entidad contratante podría, por razones medioambientales, decidir construir una sala de videoconferencia, en lugar de gastar dinero en viajes para reuniones. Del mismo modo, podría comprar helicópteros en lugar de botes salvavidas para el rescate marítimo a fin de apoyar a una industria nacional de helicópteros. La decisión sobre cómo implementar un proyecto, así como la decisión de llevarla a cabo, también puede verse influida por el deseo de desarrollar nuevos productos o servicios.

No existe una línea claramente demarcada entre el concepto de una decisión de comprar o no, como se discutió anteriormente, y la decisión sobre qué comprar ${ }_{i}$ ni entre la decisión sobre qué comprar y cómo describir esa compra en las condiciones del contrato (que se analiza a continuación). Por

53 Nota del traductor: El lector debe tener en cuenta que este artículo fue originalmente publicado pocos años después de la crisis económica del año 2008. 
lo tanto, la elección de los medios puede comprometer la funcionalidad p $_{\text {p }}$ ejemplo, las videoconferencias pueden ser inferiores a las reuniones para garantizar debates efectivos, pero pueden ser elegidas por los beneficios ambientales. Entonces, si una decisión de elegir un medio en particular, o de reducir la magnitud de un proyecto, debe clasificarse como una decisión de no contratar o una decisión sobre qué contratar es una cuestión de grado. De manera similar, si un requisito específico es meramente una función de un producto o un medio diferente para satisfacer una necesidad es también una cuestión de grado. Sin embargo, se trata de una clasificación conveniente para fines descriptivos.

\subsection{Cláusulas contractuales establecidas por la entidad estatal}

Una vez que se toma la decisión de contratar, las entidades pueden tratar de implementar objetivos sociales o ambientales a través de cláusulas contractualmente vinculantes. Estas condiciones, a menudo, pueden estar relacionadas únicamente con la ejecución del contrato. Dichas condiciones, como muchos de los otros mecanismos que se analizarán a continuación, pueden ser de varios tipos: por lo tanto, como se explicó, pueden relacionarse con medidas de consumo, producción o suministro, medidas de disposición final, o asuntos relativos a la mano de obra. Además, se pueden establecer cláusulas para promover el cumplimiento de obligaciones que no están limitados a la ejecución del contrato. Por ejemplo, que una entidad exija a sus contratistas que implementen políticas de contratación equitativa en todos sus negocios podría hacerse a través de una cláusula del contrato.

Además de establecer las condiciones contractuales que deberán cumplir todos los oferentes seleccionados, los gobiernos también suelen incluir cláusulas contractuales como parte de otros mecanismos de política. Por ejemplo, pueden exigir garantías de que el contratista en cuestión es elegible para contratos reservados (para minorías) o para criterios de adjudicación preferentes.

$\mathrm{Al}$ igual que sucede con otras condiciones contractuales, es aconsejable redactar las condiciones contractuales "horizontales" para maximizar sus beneficios al brindar a los proveedores la flexibilidad sobre cómo cumplir con los requisitos funcionales del gobierno. Por ejemplo, con una política que requiere que las empresas utilicen desempleados por largo tiempo en contratos estatales, puede no ser apropiado especificar con precisión cómo deben contratarse las personas desempleadas (ya sea como empleados, empleados de subcontratistas, etc.); dejar a los contratistas en libertad para utilizar los métodos más eficientes, especialmente dado que los contratistas probablemente tengan un mejor conocimiento del mercado, podría ser más beneficioso. Limitar las especificaciones a los requisitos funcionales puede, de hecho, tener beneficios que van más allá del contrato al fomentar la innovación. 
El enfoque de establecer las condiciones contractuales para todos los contratistas es adecuado cuando hay requisitos primordiales que tienen que cumplirse. Este será el caso, por supuesto, si las condiciones simplemente reflejan los requisitos legales existentes, como las obligaciones de pagar el salario mínimo.

Las condiciones que deben cumplir todos los contratistas pueden también ser útiles en otras situaciones en las cuales existe información confiable de que los costos adicionales para cumplir los requisitos están dentro de los límites aceptables o (menos comúnmente) cuando el gobierno no desea realizar la compra sin estos beneficios (en este caso, si el precio es demasiado alto, la compra no será aprobada). Como ya se mencionó, los costos adicionales pueden surgir tanto por el costo adicional para las empresas de cumplir con los requisitos como porque algunas empresas no pueden cumplir con los requisitos $y$, por lo tanto, no pueden participar.

Antes de establecer requisitos horizontales que van más allá de la ley, es útil que los gobiernos consideren si es preferible un enfoque alternativo. En particular, es relevante considerar la posibilidad de criterios de adjudicación sociales o ambientales (mecanismo 8). El uso de criterios de adjudicación puede permitir una evaluación más precisa de los costos, proporcionar un mecanismo para limitarlos y facilitar la mejor combinación general de beneficios sociales/ ambientales, por un lado, y de precio y otras características, por el otro.

Un posible enfoque es evaluar los costos de ciertos requisitos sociales o ambientales exigiendo o permitiendo variaciones, es decir, ofertas que proponen un enfoque diferente del sugerido en una solución "estándar", incluso porque presentan características adicionales o porque omiten ciertas características de la oferta estándar. Permitir o requerir variaciones en lugar o además de una oferta estándar puede posibilitar a los gobiernos evaluar los costos adicionales de las características adicionales u omitidas, al comparar los costos de la variación y las ofertas estándar, y tenerlos en cuenta en los criterios de adjudicación del contrato. Por ejemplo, un gobierno podría incluir un requisito para proporcionar empleo para los desempleados en una oferta estándar, pero también permitir variaciones en las ofertas sin estos beneficios sociales. Los criterios de adjudicación deberán incluir los beneficios sociales proporcionados por cada oferta, de modo que si estos superan los costos adicionales, la entidad puede adjudicar el contrato a una oferta que ofrezca el beneficio social en lugar de uno que no lo haga.

Por otro lado, a veces puede resultar apropiado exigir el cumplimiento de normas que van más allá de la ley, independientemente de todas estas consideraciones. Este es especialmente el caso de si estos estándares tienen una importancia simbólica que podría verse comprometida por un intercambio abierto entre aspectos horizontales y consideraciones comerciales, por ejemplo, cuando el gobierno busca promover el cumplimiento de ciertos estándares ambientales dando ejemplo. Incluso en este caso, sin embargo, se puede 
proporcionar cierta flexibilidad a través de la discreción que existe para hacer cumplir las condiciones en la práctica ${ }^{54}$.

Adicionalmente, los requisitos mínimos sobre características o beneficios sociales o ambientales para todos los oferentes a veces pueden combinarse con criterios de adjudicación, que otorgan puntos adicionales a aquellos productos o servicios con mejores características ambientales o sociales. Por ejemplo:

Una condición obligatoria podría requerir que todas las tecnologías de la información para una nueva biblioteca sean accesibles para usuarios sordos, ciegos y/o con discapacidad física [...]. Alternativamente, podría requerirse que un porcentaje de computadores se ajuste a las necesidades de usuarios con discapacidades, pero se otorgarán algunos puntos por extenderlo más allá del porcentaje especificado (una especificación obligatoria combinada con un criterio de adjudicación) o simplemente la accesibilidad del equipo será un factor para juzgar la oferta más ventajosa (solo un criterio de adjudicación). Se puede utilizar un criterio de adjudicación relacionado con la accesibilidad cuando la autoridad está preparada para comprometer un presupuesto fijo para la transformación de TIC y desea seleccionar el oferente que puede ofrecer la mejor relación calidad/precio o la mayor accesibilidad (según sus prioridades, expresadas en los criterios de adjudicación ${ }^{55}$.

Además de incluir condiciones contractuales para obtener beneficios sociales o ambientales específicos en el contrato (o para limitar su impacto adverso), un motivo diferente, o a menudo adicional, para tales condiciones es promover el desarrollo y la producción en masa de productos con características sociales o ambientales deseables (como se señaló, los gobiernos incluso pueden tomar la decisión inicial sobre qué proyectos emprender por este motivo). Por ejemplo, un gobierno puede decidir comprar equipos de TIC con características que los hagan accesibles a usuarios con discapacidad no solo para el beneficio de los empleados y el público que usan estos equipos, sino también para fomentar el desarrollo y la fabricación de equipos económicamente asequibles que pueden ser comprados por el sector privado ${ }^{56}$. Esta es una razón adicional para incluir características tales como los requisitos obligatorios de las especificaciones y no simplemente como criterios de adjudicación, pues esto puede ser necesario para garantizar la existencia de un mercado.

Existen diversos medios disponibles para garantizar el cumplimiento de las condiciones del contrato. En primer lugar, los recursos administrativos y judiciales de carácter contractual (contractual remedies) pueden estar disponibles (de hecho, pueden ser la motivación para incluir la obligación como parte del contrato). A menudo, los recursos y acciones son simplemente aquellos 
disponibles en virtud del derecho contractual nacional, que pueden incluir un derecho a rescindir el contrato, una orden exigiendo el cumplimiento y/o un derecho a indemnización por incumplimiento (cuya naturaleza e importe pueden depender del derecho contractual nacional). Sin embargo, los recursos y acciones contractuales generales pueden ser difíciles de ejercer: por ejemplo, una reparación por daños puede requerir un daño específico y cuantificable al gobierno, lo cual es difícil de probar por incumplimiento de las condiciones sociales, mientras que la terminación del contrato puede ser inconveniente debido a los costos y retrasos. Por esta razón, puede ser útil proporcionar consecuencias (remedies) adecuadas directamente en la legislación (como sanciones económicas especificas); esto se ha hecho en Sudáfrica para hacer cumplir los requisitos del contrato relacionados con, entre otras cosas, la participación de grupos desfavorecidos ${ }^{57}$.

Asimismo, para asegurar los beneficios de las políticas de la entidad, puede ser apropiado rechazar las ofertas que no acepten las condiciones contractuales. A menudo, las entidades estarán obligadas a hacerlo en virtud de las leyes de contratación que prohíben la aceptación de ofertas que no acepten las condiciones obligatorias.

Una entidad también puede considerar excluir incluso a los oferentes que estén dispuestos a aceptar las cláusulas, cuando considere que el oferente no puede cumplir, o no cumplirá realmente. Esto puede ser importante debido a las dificultades prácticas que pueden existir al ejercer los recursos legales para remediar un incumplimiento real. Sin embargo, la posibilidad de rechazar a los contratistas por adelantado aumenta la discreción.

Se puede observar que la directiva de contratación pública (Directiva 2004/18/CE) ${ }^{58}$, que limita la discrecionalidad para disuadir y monitorear la discriminación, prohíbe a un comprador público excluir a un contratista simplemente porque el comprador cree que el contratista no puede o no quiere cumplir con las condiciones laborales del contrato, como los requisitos para contratar a personas desempleadas. Esto es debido a una preocupación sobre la mayor discreción que esto conlleva. Esta posición es criticada por elevar los aspectos comerciales en la contratación por encima de los aspectos sociales/ ambientales, al proporcionar una aplicación más estricta para los primeros que para los segundos. Esto es así, pues generalmente se permite a las entidades excluir a los contratistas que creen que no pueden cumplir con elementos "comerciales" del contrato como los estándares de calidad ${ }^{59}$.

57 Véase Preferential Procurement Regulations 2001, en relación con Preferential Procurement Policy Framework Act, n. ${ }^{\circ} 5$ of 2000, Regulation 15.

58 Nota del traductor: El lector debe tener en consideración que esta directiva fue reemplazada por la directiva 2014/24/UE.

59 Sue Arrowsmith, "Application of the EC Treaty and Directives to Horizontal Policies: a Critical Review", óp. cit., sección 8.1.4, Christopher MCCRUDDEn, óp. cit., capítulos 16 y 17. 


\subsection{Agrupamiento y MOMENTO DE LAS ÓRDENES}

Los gobiernos también pueden implementar políticas horizontales a través de la forma en que colocan pedidos en el mercado, bien sea a través de la manera en que agrupan sus órdenes o el momento de esas órdenes. Esta es una estrategia utilizada a menudo para promover la participación de las pequeñas y medianas empresas (PYMES) en la contratación pública ${ }^{60}$. Por ejemplo, una gran orden se puede dividir en lotes separados otorgados al mismo tiempo o distribuidos a lo largo del tiempo para permitir licitaciones por montos pequeños.

Dichos mecanismos pueden buscar mejorar la relación calidad-precio mediante la ampliación del mercado para incluir más empresas, tanto más pequeñas como más grandes. Sin embargo, también pueden buscar específicamente apoyar el desarrollo de las PYMES como un objetivo de política industrial. En este último caso, los costos son principalmente administrativos, es decir, los costos adicionales de tener y administrar un gran número de contratos más pequeños en lugar de un contrato grande (si se desea, estos costos adicionales pueden tenerse en consideración en la evaluación de la oferta como un criterio de adjudicación). Sin embargo, los gobiernos pueden adoptar tales mecanismos incluso cuando implican precios más altos u otra pérdida. A manera de ejemplo, las entidades pueden agrupar obras en pequeñas cantidades con procedimientos de adjudicación separados, aunque se acepte que esto puede conducir a precios más altos al disuadir a las empresas más grandes de licitar; o pueden limitar la cantidad de trabajo que puede hacer una sola empresa para promover la participación de las PYMES, aunque esto puede significar rechazar las ofertas que ofrecen mejores precios.

\subsection{Contratos Reservados (SET-ASIDES)}

Otro mecanismo para implementar políticas horizontales es limitar la participación a un grupo en particular. Este enfoque se ha utilizado, por ejemplo, para apoyar talleres para presos y personas discapacitadas. Por ejemplo, las directivas de la UE de $2004^{[61]}$ ahora permiten expresamente el uso de contratos reservados para talleres que ofrecen empleo a personas con discapacidad, como excepción a una prohibición general de contratos reservados ${ }^{62}$. Los contratos reservados también se utilizan a menudo para proporcionar oportunidades económicas

60 Por ejemplo: European Commission, Staff Working Document of 25.6.2008, European Code of Best Practices Facilitating Access by SMEs to Public Procurement Contracts, (SEC (2008) 2193), 2008. Disponible en línea http://ec.europa.eu.

61 Nota del traductor: Nuevamente se advierte al lector que estas directivas fueron reemplazadas, luego de la publicación del artículo original en inglés, por la "ola" de directivas de 2014. 
a grupos étnicos desfavorecidos y PYMES. Son una característica importante de las políticas del gobierno de Estados Unidos para promover las pequeñas empresas, en particular las que son propiedad de personas desfavorecidas ${ }^{63}$.

El gobierno puede favorecer las políticas de contratos reservados tanto por su alta visibilidad-mediante resultados específicos en los contratos adjudicados a los beneficiarios-como porque la adjudicación garantizada e inmediata de contratos puede producir resultados económicos rápidos. Los gobiernos pueden hacer los contratos reservados más efectivos a través de términos contractuales, como una garantía de que el contratista es elegible para la reserva.

Sin embargo, reservar contratos para ciertos grupos también puede implicar un número significativo de costos adicionales ${ }^{64}$.

Así, los costos pueden surgir debido a la reducción de la competencia, combinada con el hecho de que los grupos beneficiarios pueden no ser tan competitivos como los excluidos. Los gobiernos pueden estar dispuestos a pagar costos adicionales, o pueden intentar eliminarlos permitiendo el uso de contratos reservados solo cuando se puede operar en condiciones comerciales (commercial terms). Al igual que con las políticas implementadas a través de las cláusulas del contrato, el procedimiento de selección en sí no proporcionará información precisa sobre los costos adicionales en los cuales se incurrirá.

Sin embargo, los gobiernos pueden, hasta cierto punto, retener los beneficios de la competencia al utilizar un procedimiento de selección competitivo y luego permitir que el mejor postor del grupo beneficiario ejecute el contrato reservado solamente si dicho oferente iguala los mejores términos ofertados (el enfoque de ofrecer de nuevo offer back approacb). Este mecanismo fue usado una vez por el gobierno británico para importantes contratos adjudicados para apoyar talleres para personas con discapacidad (el esquema de "proveedores prioritarios"), aunque finalmente fue abandonado debido a las restricciones impuestas por la legislación de la $\mathrm{UE}^{65}$.

Otro problema con los contratos reservados es que los grupos beneficiarios pueden tener incentivos insuficientes para volverse competitivos, y si un objetivo de la política es desarrollar industrias o empresas que sean competitivas en un mercado más amplio, los contratos reservados pueden ser contraproducentes. Las reservas de contratos también pueden introducirse o mantenerse como resultado de la presión política cuando no son necesarios, lo que genera costos sin beneficios concretos.

En algunos casos, en particular cuando se promueve un "campeón nacional" o se asignan contratos estratégicamente para mantener la competencia, los

63 John Cibinic y Ralph Nash, óp. cit., capítulo 10.

64 International Trade Centre, Public Procurement and Smes. Improving Access of Small and Medium Enterprises to Public Procurement: Experiences of Selected Countries, 2000. Disponible en línea http://www.intracen.org. 
contratos se pueden reservar no solo para un grupo limitado sino para empresas específicas. También pueden asignarse a empresas específicas sin competencia en otros casos: bajo el antiguo esquema del Reino Unido para apoyar talleres para personas con discapacidad, el gobierno asignó órdenes de bajo valor a estos talleres sin competencia, cuando el gobierno estaba convencido de que el suministro se hacía en términos comerciales ${ }^{66}$.

\subsection{EXCLUSIÓN PARA CONTRATAR POR INCUMPLIMIENTO}

DE LAS POLÍTICAS GUBERNAMENTALES

Otro mecanismo importante para implementar políticas horizontales es la exclusión de los contratos. La exclusión o la amenaza de exclusión se puede utilizar para reforzar el cumplimiento de las políticas del gobierno y/o para penalizar violaciones pasadas.

Para poner en contacto este mecanismo con la primera distinción de nuestra taxonomía, podemos notar que al igual que con muchos otros utilizados en la contratación, tales como las cláusulas contractuales, las exclusiones pueden reforzar el cumplimiento de normas generales no limitadas a contratistas del gobierno, como las de la ley penal. La exclusión obligatoria de la UE para las empresas condenadas por ciertos delitos y la política de Irlanda del Norte de excluir a empresas que violan las obligaciones de no discriminar por motivos de creencias religiosas o políticas, ambas discutidas anteriormente, son ejemplos de esto.

Las exclusiones también se pueden utilizar, sin embargo, para reforzar normas establecidas únicamente para los contratistas del gobierno. Este, como se señaló, es el caso de la política de Estados Unidos de excluir a los contratistas que no implementan políticas de reclutamiento de personal que promuevan la igualdad en el lugar de trabajo.

Con respecto a la segunda distinción de la taxonomía, las exclusiones (como en el caso de algunos otros mecanismos) pueden limitarse a respaldar el cumplimiento de las normas en la ejecución del contrato adjudicado. Por lo tanto, los gobiernos pueden excluir a una empresa simplemente para asegurarse de que se adjudique el contrato solo a un proveedor que efectivamente pueda cumplir ciertas condiciones contractuales (ya sea en materia de consumo, suministro o producción, disposición final, o mano de obra contratada); por ejemplo, una cláusula que exija que la electricidad que suministre el contratista en la ejecución del contrato provenga de fuentes renovables.

Sin embargo, las exclusiones también pueden utilizarse como una herramienta para garantizar el cumplimiento de requisitos que no se limitan a la ejecución del contrato, como con las disposiciones de exclusión obligatorias de la UE 
(que exigen la exclusión por todas las condenas anteriores) y las políticas de Estados Unidos que promueven igualdad en el lugar de trabajo. El gobierno también puede usar la exclusión como una sanción por el incumplimiento en contratos públicos anteriores, como la violación de los términos contractuales sobre condiciones de trabajo justas. El incumplimiento de obligaciones en el pasado puede, por supuesto, proporcionar evidencia de la incapacidad para cumplir en el futuro, y el incumplimiento pasado podría invocarse para excluir por este motivo, así como usarse a modo de sanción por las violaciones pasadas.

Con frecuencia, un gobierno puede encontrar conveniente usar tanto las exclusiones como las condiciones contractuales: cuando un contrato de obra incluye obligaciones para involucrar a desempleados de larga duración en el contrato, tanto la amenaza de exclusión de contratos futuros como la existencia de acciones contractuales por incumplimiento pueden ayudar a inducir el cumplimiento.

Las exclusiones pueden tener como resultado precios más altos y/o un compromiso de calidad, tanto porque limitan la competencia como porque los contratistas que compiten pueden pasar los costos de cumplimiento al gobierno. Al igual que con los contratos reservados y las condiciones contractuales, los costos precisos pueden ser difíciles de evaluar. Además, la discreción involucrada puede crear un margen para el abuso: por ejemplo, se puede abusar de las exclusiones para excluir a las empresas que son la competencia de un proveedor favorecido.

Sin embargo, las exclusiones pueden ser útiles. En primer lugar, permiten que los gobiernos trabajen estrechamente con un grupo limitado de empresas de manera continua para mejorar las prácticas, por ejemplo, en el reclutamiento de personal. En segundo lugar, al igual que con las condiciones contractuales, pueden usarse para reforzar el cumplimiento de normas legales existentes, como prohibiciones de corrupción u otras normas (incluidas normas sobre derechos humanos) cuando un análisis explícito costo/beneficio a través de criterios de adjudicación parezca inapropiado debido a la dimensión moral. $\mathrm{Al}$ igual que con las cláusulas del contrato, el equilibrio de costos y beneficios también puede ocurrir en la práctica a través del ejercicio de la discreción sobre si se excluye o no. Incluso las exclusiones obligatorias suelen estar sujetas a excepciones por razones de interés público, como es el caso de las exclusiones obligatorias de la UE para determinadas condenas penales. El uso de esas excepciones podría potencialmente estar basado en consideraciones de costos (aunque no está claro que esto esté permitido bajo las exclusiones obligatorias de la UE mencionadas anteriormente) $)^{67}$. 


\subsection{INVITACIONES PREFERENCIALES PARA LICITAR}

En algunos procedimientos, las entidades pueden invitar a presentar ofertas solamente a un número limitado de empresas, por ejemplo, cuando los costos de evaluar muchas ofertas superan los beneficios de una mayor competencia, como lo permite el procedimiento de licitación restringida en virtud de la Ley Modelo sobre la Contratación Pública de la Comisión de las Naciones Unidas para el Derecho Mercantil Internacional (CNUDMI) ${ }^{68-69}$ Las consideraciones horizontales podrían entonces tenerse en cuenta al decidir a cuáles empresas invitar.

Es más probable que estas consideraciones se refieran a las características de los contratistas, por ejemplo, preferencias para invitar a empresas de regiones pobres o talleres para personas con discapacidad. Sin embargo, en teoría también podrían relacionarse con el desempeño de las empresas para cumplir con ciertos estándares, como los de reclutamiento justo de personal, o usarse a favor de firmas que se considere que ofrecen un mejor desempeño social o ambiental. Sin embargo, al igual que con los criterios de adjudicación, las preferencias pueden considerarse inadecuadas para abordar el cumplimiento con normas externas o con políticas basadas en principios morales (como el uso de trabajo infantil).

El uso de este mecanismo no tiene por qué afectar otros aspectos de la contratación, como el precio o la calidad, si solamente se utiliza cuando las empresas invitadas son iguales en todos los demás aspectos al resto de empresas en el mercado. Sin embargo, aún pueden existir costos administrativos o de otro tipo. Además, la relación calidad-precio también puede verse afectada si algunas empresas se abstienen de participar debido a la reducción en la posibilidad de ser invitadas a licitar.

\subsection{Criterios de adjudicación}

Otro enfoque común para implementar políticas horizontales es a través de criterios de adjudicación, es decir, considerando los méritos relativos de ofertas con respecto a sus beneficios sociales o ambientales al decidir cuál propuesta aceptar.

Los criterios de adjudicación, como las condiciones del contrato, a menudo se limitarán a la ejecución del contrato. Como ocurre con muchos otros mecanismos, pueden referirse a medidas de consumo (por ejemplo, preferencia por autobuses accesibles para sillas de ruedas), de producción o suministro (como

69 En la actualidad existe una nueva versión de la Ley Modelo sobre la Contratación Pública de la CNUDmi. Se trata de la Ley Modelo de 2011. 
preferencia por productos fabricados con materiales reciclados), de disposición final (por ejemplo, preferencia por ofertas que ofrecen devolución de los productos para reciclaje) o de mano de obra (por ejemplo, una preferencia por ofertas que ofrecen trabajo a desempleados de larga duración). Los criterios relacionados con los asuntos de la fuerza laboral se han utilizado en Irlanda del Norte, donde el gobierno emprendió recientemente un proyecto piloto para examinar los costos y el beneficio de dichos criterios. En el marco de este proyecto, los oferentes en los proyectos seleccionados debían presentar un plan de empleo para la contratación de personas desempleadas durante más de tres meses. La calidad del plan se tuvo en cuenta como un criterio de adjudicación, aunque solo cuando otros aspectos de las ofertas eran iguales ${ }^{70}$. El gobierno de Sudáfrica también ha utilizado ampliamente criterios de adjudicación para promover el desarrollo de grupos desfavorecidos ${ }^{71}$.

Los criterios de adjudicación también pueden, como muchos otros mecanismos, relacionarse con la conducta de los oferentes más allá del contrato, tales como los méritos de las políticas de diferentes empresas para combatir la discriminación en la contratación de personal en general. Y también pueden referirse a las características de la empresa en sí, como si es propiedad de una minoría étnica desfavorecida o si se encuentra en una región pobre, o en qué medida el proveedor puede ofrecer compensaciones (offsets) fuera del contrato.

Una vez más, los criterios de adjudicación no siempre son adecuados para reforzar el cumplimiento de la ley en general, pues parece inapropiado ponderar abiertamente el cumplimiento de la ley por parte del contratista con los costos u otras consideraciones.

Se pueden distinguir varios tipos de preferencias en la etapa de adjudicación, de acuerdo con la forma en que se implementan, de la siguiente manera:

a. Un enfoque es una preferencia de precio fijo para las ofertas que cumplen con ciertos criterios mínimos. Un ejemplo podría ser una preferencia de precio del $10 \%$ para productos fabricados con materiales reciclados o para empresas que puedan cumplir los requisitos establecidos para proporcionar trabajo a los desempleados de larga duración. Como se explicó anteriormente, una entidad podría proporcionar un programa de este tipo como estándar en las ofertas, pero también permitir a las empresas presentar ofertas variantes que no incluyan dicho programa, y luego podría ponderar los costos y beneficios de cada una, utilizando un criterio de adjudicación que incluya ambos aspectos financieros y beneficios sociales.

b. Otro enfoque es variar la preferencia de acuerdo con la medida en que una empresa ofrece beneficios horizontales, por ejemplo, otorgando un grado

70 Andrew Erridge y Sean Hennigan, "Public Procurement and Social Policy in Northern Ireland: The Unemployment Pilot Project", Advancing Public Procurement: Practices, Innovation and Knowledge-Sharing, Boca Raton: PrAcademics Press, 2006, pp. 280-303.

71 Phoebe Bolton, óp. cit. 
de preferencia ascendente de acuerdo con la cantidad de personas desempleadas que el oferente contratará. Sudáfrica ha seguido este enfoque al establecer diferentes preferencias de acuerdo con el grado en que las ofertas involucran a ciertos grupos desfavorecidos en el contrato ${ }^{72}$

c. Finalmente, al igual que con las invitaciones preferenciales para licitar, los beneficios horizontales podrían considerarse solamente cuando las ofertas sean iguales después de aplicar otros criterios de adjudicación. Este fue el adoptado en el proyecto piloto de Irlanda del Norte sobre el desempleo mencionado anteriormente. Sin embargo, esto puede tener un impacto limitado ya que solo en raras ocasiones los otros aspectos serán iguales. Aparentemente fue elegido en Irlanda del Norte porque el gobierno consideró que la legislación de la UE permite los criterios de adjudicación de "mano de obra" solo cuando las ofertas son iguales, aunque esta es una interpretación controvertida de la ley ${ }^{73}$.

Como ya se mencionó, los criterios de adjudicación se pueden combinar con otros enfoques, como establecer requisitos mínimos como condiciones contractuales.

El uso de criterios de adjudicación en lugar de otros métodos, tales como exclusiones o condiciones contractuales establecidas para todos los participantes, puede proporcionar un mecanismo para evaluar el costo adicional preciso de las políticas horizontales, que se reflejará en el precio y otros términos de las ofertas $;$ factores que pueden ser más confiable que las propias estimaciones de la autoridad (aunque hemos visto que los costos de las políticas implementadas a través de las condiciones del contrato a veces se pueden establecer mediante el uso de variaciones).

Esta aproximación también proporciona un mecanismo para contener los costos dentro de los parámetros establecidos de antemano, a través de la ponderación de los criterios. Por ejemplo, una ponderación del 10\% para los criterios sociales establece límites claros en la proporción del valor del contrato que se dedicará a los beneficios sociales o ambientales. Los criterios de adjudicación también permiten permutaciones variables de características sociales/ambientales y comerciales, de acuerdo con las cuales ofrece la mejor relación calidad precio, dada la ponderación que se le asigna a cada una (esto se aplica cuando la autoridad utiliza el enfoque de preferencia variable descrito anteriormente). Por todas estas razones, los criterios de adjudicación pueden preferirse a otros mecanismos.

72 Ibíd.

73 Sue Arrowsmith, "Application of the EC Treaty and Directives to Horizontal Policies: A Critical Review", Social and Environmental Policies in EC procurement Law: New Directives and New Directions, Cambridge: Cambridge University Press, 2009, pp. 147-248. 
El impacto de los criterios de adjudicación variará de acuerdo con la competitividad y la estructura de mercados particulares, pero se pueden hacer algunas observaciones generales sobre los potenciales $\operatorname{costos}^{74}$.

Un problema con las preferencias en la selección para determinadas empresas, como las minorías desfavorecidas, es que, al igual que algunos otros mecanismos, aunque no necesariamente en la misma medida, pueden implicar premios a empresas menos eficientes. Es más probable que estas empresas sobrevivan cuando el gobierno divide el mercado con un margen de preferencia de precio que cuando divide el mercado con contratos reservados. En los mercados imperfectos, los criterios de adjudicación pueden alentar a las empresas a cobrar al gobierno un precio más alto de lo que cobran al sector privado. Al igual que con los contratos reservados, también existe el peligro de que las preferencias se apliquen cuando no es necesario, lo que genera costos sin los correspondientes beneficios; esto parece haber sucedido con contratos reservados para pequeñas empresas en Estados Unidos (véase, por ejemplo, la revisión del uso de las preferencias de precios en ciertas medidas de contratación de Estados Unidos en el Federal Register, vol. 63 n. ${ }^{\circ} 125$ al 35.713). Sin embargo, tales políticas al menos proporcionan algunos incentivos para que los oferentes beneficiados funcionen más eficientemente para ganar contratos.

$\mathrm{Al}$ igual que al diseñar las cláusulas contractuales para una máxima eficacia, los criterios de adjudicación deberían centrarse en los resultados y no ser excesivamente prescriptivos al establecer cómo se deben lograr los beneficios. Del mismo modo que puede resultar útil no especificar con precisión cómo deben emplearse las personas desempleadas en un contrato cuando su empleo se especifica como una condición contractual (ya sea como empleados, empleados de subcontratistas, etc.), las empresas pueden quedar libres para proponer cómo emplear a las personas desempleadas cuando esto se tiene en cuenta como un criterio de adjudicación.

Los beneficios horizontales ofrecidos en las ofertas se pueden incluir como obligaciones contractuales. Cuando una empresa ofrece contratar a personas desempleadas de larga duración, por encima de cualquier mínimo requerido, este compromiso puede incluirse como un término del contrato, con lo que los recursos y acciones contractuales (remedies) estarán disponibles para garantizar el cumplimiento de esas obligaciones. Con los criterios de adjudicación basados en el carácter del contratista, por ejemplo, ser propiedad de un grupo desfavorecido, el gobierno puede hacer efectiva la política incluyendo una garantía contractual de que el contratista efectivamente cumple con los términos de la preferencia. 


\subsection{Medidas para mejorar el acceso a los contratos estatales}

Un enfoque final comprende medidas para facilitar el acceso a contratos para ciertos grupos sin alterar las condiciones de competencia (es decir, sin proporcionar un trato favorable en la competencia) y sin ajustar los requerimientos del gobierno (por ejemplo, características del producto o el momento de contratar). Los gobiernos pueden proporcionar capacitación sobre procedimientos de selección a las PYMES o a aquellos pertenecientes a grupos minoritarios desfavorecidos para ayudarlos a conocer y tener acceso a oportunidades, pero no dar un trato preferencial en el procedimiento de selección. Las medidas para simplificar el sistema de contratación y reducir las cargas, por ejemplo, al permitir que los contratistas accedan a contratos en todo el gobierno al completar un solo cuestionario, también suelen tener como objetivo facilitar la participación de proveedores más pequeños y desfavorecidos.

Puede haber costos en la administración y el financiamiento de tales programas. Sin embargo, es poco probable que se produzca un impacto adverso sobre la relación calidad-precio en la propia selección, de hecho, a menudo es un objetivo mejorar la relación calidad-precio a través del fomento de una competencia más amplia.

\section{CONCLUSIONES}

En este artículo se ha presentado una taxonomía de políticas horizontales en la contratación pública basada en tres distinciones clave que son relevantes independientemente del tema de la política en cuestión. El primero es una distinción entre las políticas que se limitan a garantizar el cumplimiento de los requisitos legales y las que van más allá, el segundo es una distinción entre las medidas aplicadas solo al contrato adjudicado, que se distinguen de aquellas que van más allá; y, en tercer lugar, se identificaron nueve mecanismos diferentes a través de los cuales se aplican las políticas horizontales en la contratación.

Como se explicó brevemente, los diferentes enfoques y mecanismos utilizados presentan diversos problemas y desafíos en términos de transparencia, legitimidad y efectividad, así como en términos de impacto en los intereses de los contratistas y en el comercio internacional. Las cuestiones legales y de política que surgen en esta área son desafiantes y no son fáciles de responder. Sin embargo, una comprensión de las diferentes variaciones y matices de las políticas horizontales puede ayudar a iluminar las elecciones que se realizan y, de este modo, facilitar el buen desarrollo de las leyes y las políticas en este campo. 


\section{BIBLIOGRAFÍA}

Anechiarico, Frank y James Jacobs. "Purging Corruption from Public Contracting: The Solutions are Now Part of the Problem", New York Law School Law Review, vol. 40, New York: New York Law School, 1995.

Arrowsmith, Sue. "Application of the EC Treaty and Directives to Horizontal Policies: A Critical Review", in Social and Environmental Policies in EC procurement Law: New Directives and New Directions, Cambridge: Cambridge University Press, 2009.

Arrowsmith, Sue. Government Procurement in the World Trade Organization, London: Kluwer Law International, 2003.

Arrowsmith, Sue. "The EC Procurement Directives, National Procurement Policies and Better Governance: The Case for a New Approach", European Law Review, n. ${ }^{\circ}$ 1, London: Sweet \& Maxwell, 2002.

Arrowsmith, Sue. The Law of Public and Utilities Procurement, London: Sweet \& Maxwell, 1996.

Arrowsmith, Sue. The Law of Public and Utilities Procurement, 2. ${ }^{\text {a }}$ ed., London: Sweet \& Maxwell, 2005.

Arrowsmith, Sue y Peter Kunzlik (eds.). Social and Environmental Policies in EC procurement Law: New Directives and New Directions, Cambridge: Cambridge University Press, 2009.

Arrowsmith, Sue, John Linarelli y Don Wallace. Regulating Public Procurement: National and International Perspectives. London: Kluwer Law International, 2000.

Bailey, Stephen. "Judicial Review of Contracting Decisions.", Public Law, n. o 3, London: Sweet \& Maxwel, 2000.

Bercusson, Brian. The Fair Wages Resolutions. London: Mansell, 1978.

Bolton, Phoebe. "An Analysis of the Preferential Procurement Legislation of South Africa", Public Procurement Law Review, vol. 16, n. ${ }^{\circ}$ 1, London: Sweet \& Maxwel, 2007.

Boyle, Rosemary. "Disability Issues in Procurement", in Social and Environmental Policies in EC procurement Law: New Directives and New Directions, Cambridge: Cambridge University Press, 2009.

Carr, John. New Roads to Equality: Contract Compliance for the United Kingdom?, London: The Fabian Society, 1987.

Cibinic, John y Ralph Nash. Formation of Government Contracts, 3. ${ }^{\circ}$ ed., Washington: Government Contract Program, George Washington University, 1998. 
Cleveland, Sara H. "Norm Internalization and U.S. Economic Sanctions", Yale Journal of International Law, vol. 26, n. ${ }^{\circ}$ 1, New Haven: Yale University, 2001.

Daintith, Terence. "Regulation by Contract: The New Prerogative", Current Legal Problems, vol. 32, Oxford: Oxford University Press, 1979.

Department for Environment, Food and Rural Affairs. UK Government Sustainable Development Action Plan (November 2007 - November 2009), 2007. Disponible en línea http://www.defra.gov.uk/sustainable/government/publications/pdf/ SustainableProcurementActionPlan.pdf.

Erridge, Andrew y Sean Hennigan. "Public Procurement and Social Policy in Northern Ireland: The Unemployment Pilot Project", Advancing Public Procurement: Practices, Innovation and Knowledge-Sharing, Boca Raton: PrAcademics Press, 2006.

European Commission. Interpretative Communication on the Community Law Applicable to Public Procurement and the Possibilities for Integrating Social Considerations into Public Procurement, (COM (2001) 566 final), 2001. Disponible en línea http://ec.europa.eu.

European Commission. Staff Working Document of 25.6.2008, European Code of Best Practices Facilitating Access by smes to Public Procurement Contracts, (SEC (2008) 2193), 2008. Disponible en línea http://ec.europa.eu.

Government of South Africa. Green Paper on Public Sector Procurement (GN no.691, GG17928 of 14 April 1997). UnCitral (1994). UnCitral Model Law on Procurement of Goods, Construction and Services, 1997. Disponible en línea http://www.uncitral.org/uncitral/en/uncitral_texts/procurement_infrastructure/1994Model.html.

International Trade Centre. Public Procurement and smes. Improving Access of Small and Medium Enterprises to Public Procurement: Experiences of Selected Countries, 2000. Disponible en línea http://www.intracen.org.

Kelman, Steve. Procurement and Public Management. Washington: AeI Press, 1990.

Kunzlik, Peter. "Environmental Issues in International Procurement", in Public Procurement: Global Revolution, London: Kluwer Law International, 1998.

KunZlik, Peter. "The Procurement of 'Green' Energy", in Social and Environmental Policies in EC procurement Law: New Directives and New Directions, Cambridge: Cambridge University Press, 2009.

Letchmiah, Deen. "The Process of Public Sector Procurement in South Africa", Public Procurement Law Review, vol. 8, n. ${ }^{\circ}$, London: Sweet \& Maxwel, 1999. 
Linarelli, John. "Economic Sanctions and the US Supreme Court: Crosby v National Foreign Trade Council", Public Procurement Law Review, vol. 10, n. ${ }^{\circ}$ 3, London: Sweet \& Maxwel, 2001.

McCrudden, Christopher. Buying Social Justice: Equality, Government Procurement, \& Legal Change, Oxford: Oxford University Press, 2007.

Morris, Peter. "Legal Regulation of Contract Compliance: an Anglo-American Comparison", Anglo-American Law Review, vol. 19, n. ${ }^{\circ}$ 2, Chichester: Justice of Peace, 1990.

Priess, Hans Joachim y Christian Pitschas. "Secondary Criteria and Their Compatibility with EC and wTo Procurement - The Case of the German Scientology Declaration", Public Procurement Law Review, vol. 9, n. ${ }^{\circ}$ 4, London: Sweet \& Maxwel, 2000.

Schooner, Steven. "Fear of Oversight: The Fundamental Failure of Businesslike Government", American University Law Review, vol. 50, n. ${ }^{\circ}$ 3, Washington: American University Washington College of Law, 2001.

Ssennoga, Francis. "Examining Discriminatory Procurement Practices in Developing Countries", Journal of Public Procurement, vol. 6, n. ${ }^{\circ}$ 3, Bingley: Emerald Publishing Limited, 2006.

Vogel, David. The Market for Virtue: The Potential and Limits of Corporate Social Responsibility, Washington: Brookings Institution Press, 2005.

Watermeyer, Ron B. "Facilitating Sustainable Development through Public and Donor Procurement Regimes", Public Procurement Law Review, vol. 13, n. ${ }^{\circ}$ 1, London: Sweet \& Maxwel, 2004.

Williams, Sope. "Co-ordinating public procurement to support EU objectives- a First Step? The Case of Exclusions for Serious Criminal Offences", Social and Environmental Policies in EC procurement Law: New Directives and New Directions, Cambridge: Cambridge University Press, 2009.

Wittie, Patricia. "Transnational Concerns: Domestic Preferences", Public Procurement Law Review, vol. 11, n. ${ }^{\circ}$ 2, London: Sweet \& Maxwel, 2002.

World BANK. Guidelines for Procurement under IBRD Loans and IDA Credits, 2004. Disponible en línea www.worldbank.org.

Yukins, Christopher. "Making Federal Information Technology Accessible: A Case Study in Social Policy and Procurement", Public Contract Law Journal, vol. 33, n. ${ }^{\circ} 2$, Washington: American Bar - George Washington University Law School, 2004. 